\title{
Checkpoint inhibitors in primary mediastinal B-cell lymphoma: a step forward in refractory/relapsing patients?
}

\author{
Monica Balzarotti, Armando Santoro \\ Humanitas Cancer Center, Rozzano, Italy \\ Correspondence to: Monica Balzarotti, MD. Via Alessandro Manzoni, 56, 20089 Rozzano, MI, Italy. Email: monica.balzarotti@cancercenter.humanitas.it. \\ Provenance and Peer Review: This article was commissioned by the editorial office, Annals of Translational Medicine. The article did not undergo \\ external peer review. \\ Comment on: Armand P, Rodig S, Melnichenko V, et al. Pembrolizumab in Relapsed or Refractory Primary Mediastinal Large B-Cell Lymphoma. J \\ Clin Oncol 2019;37:3291-9.
}

Submitted Feb 24, 2020. Accepted for publication Mar 25, 2020.

doi: $10.21037 / \mathrm{atm} .2020 .04 .06$

View this article at: http://dx.doi.org/10.21037/atm.2020.04.06

Armand and coauthors recently reported the exciting results on the use of pembrolizumab, a first-class immune anti-programmed death-1 (PD-1) checkpoint inhibitor $(\mathrm{CP})$, in pretreated primary mediastinal B-cell lymphoma (PMBCL) (1). They analyzed two consecutive cohorts of patients, namely a subgroup of the phase $1 \mathrm{~b}$ KEYNOTE-013 trial and of the phase II KEYNOTE-170 trial, involving a total of 74 patients. Enrolled subjects had received three previous lines of therapy (median value) and one-third of them had undergone transplantation. Objective response was reported in $45 \%$ (33\% complete responses) and $48 \%$ (13\% complete responses) of patients in the two cohorts, respectively. Median duration of response could not be reached in the first group and was $\geq 12$ months in approximately $75 \%$ of cases in the second group. At 12 months, the rate of progression-free survival was $47 \%$ and $38 \%$ in the two groups, respectively; corresponding figures for overall survival were $65 \%$ and $58 \%$, respectively. No special toxicity concerns were reported; $15 \%$ of patients experienced grade 3 or 4 toxicities. The most common adverse event was neutropenia, whereas specific CPi-related immune-mediated events occurred in two cases (one grade 4 myositis and one grade 3 pneumonitis).

Overall, PMBCL accounts for 3\% of all non-Hodgkin lymphomas (NHLs) and for 10\% of all diffuse large B-cell lymphomas (DLBCLs). Although rare, its unique clinical presentation and biological/molecular assessment have stimulated several analyses and papers. Therefore, it is presently one of the best and most deeply known subsets of lymphoma $(2,3)$.
The resemblance of PMBCL with Hodgkin lymphoma (HL) has been described since the last century (4), when an intense debate arose on whether PMBCL should be treated upfront by an HL- or an NHL-oriented approach. Later, when NHL therapy became the dominant therapeutic strategy, an even stronger debate was focused on the choice of chemotherapy regimen, with a more intense MACOP-B $(5,6)$ and derived regimens being considered as "the winners". The rituximab era changed this point of view, as its addition to any chemotherapy regimen in B-cell lymphomas annulled the difference among different single regimens and improved outcomes (7). Currently, approximately $80 \%$ of all cases are cured by first-line treatment.

On the other hand, even during this long period of debate on the choice of first-line treatment, a general agreement remained on the second-line treatment for patients failing or relapsing upon upfront therapy. Even in this setting, the treatment strategy is in line with experiences in DLBCL and consists of secondline chemotherapy followed by autologous stem cell transplantation in chemotherapy-sensitive subjects (8). In fact, this standard approach has historically obtained a worse outcome in PMBCL $(9,10)$ compared with other types of DLBCL. These disappointing results can be attributed to two main reasons: first, approximately two-thirds of recurrent PMBCLs are primary refractory or early relapse, defined as cases who progress during first-line treatment, or those who do not achieve complete remission, or relapse early after the end of upfront therapy (8). Second, both the 
Table 1 Outcome of treatment strategies in relapsing-remitting PMBCL

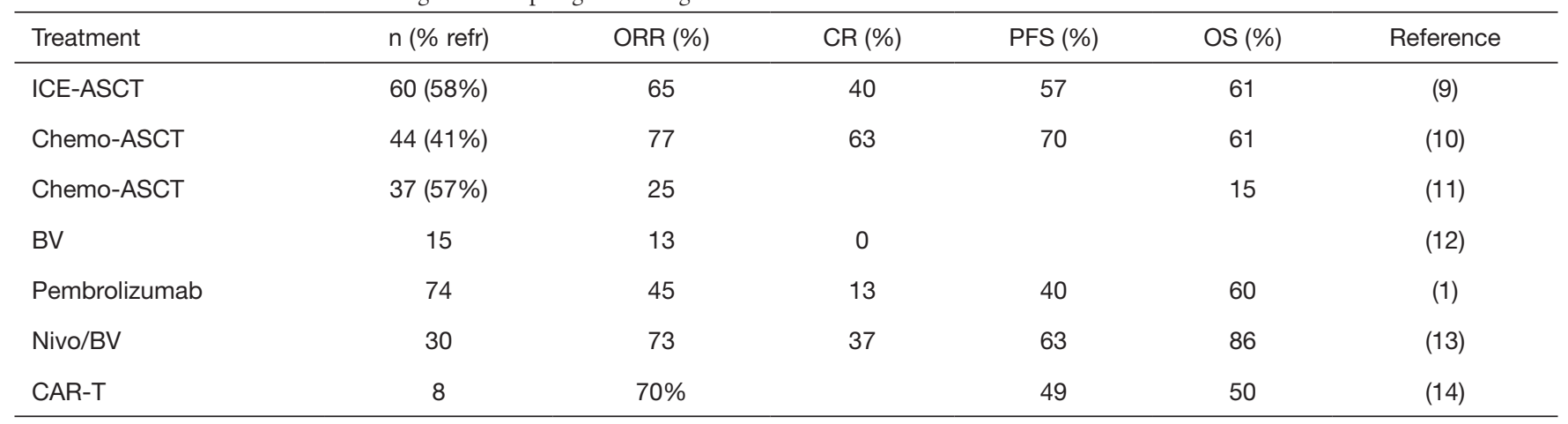

PMBCL, primary mediastinal B-cell lymphoma; ASCT, autologous stem cell transplantation; BV, brentuximab vedotin; CR, complete remission; ICE, ifosfamide, carboPt, etoposide; Nivo, nivolumab; ORR, overall response rate; OS, overall survival; PFS, progression-free survival; Refr, refractory.

overall and complete remission rates are somewhat lower than in classical DLBCL (Table 1), and therefore a lower proportion of patients can proceed with autologous stem cell transplantation $(9,10)$. Remarkably, due to the rarity of the disease and the relatively low incidence of relapse, the above considerations are extrapolated from retrospective and often small series of patients, or from subgroup analyses of randomized trials on DLBCL.

Overall, $20 \%$ of subjects with PMBCL will fail first-line therapy and more than half of them will also be refractory to second-line chemotherapy, and therefore an unmet clinical need exists for this setting of patients.

In most hematological malignancies, biological drugs targeting specific biological mechanisms are showing very promising results (15).

PMBCL harbors several molecular alterations that may represent the target of novel therapies (16-18). HL and PMBCL present a similar gene-expression profile, harboring genetic alterations of chromosome 9p (16-18). In particular, 9p24.1 is amplified in $53-70 \%$ of PMBCL and $30-40 \%$ of HLs, but only rarely in DLBCL (19). In PMBCL and HL, amplification of 9p24.1 was observed with both increased PD-L1/2 and cell-surface protein expression. PD-L1 is expressed in $30-80 \%$ of PMBCL cases, and the expression of PD-L2 protein is markedly increased in PMBCL. By way of comparison, $70-87 \%$ of classical HL and $31-57 \%$ of DLBCL express PD-L1 (20). According to the above, genetic alterations at 9p24.1 associates with increased expression of PD-1 ligands, thus conferring sensitivity to $\mathrm{CPi}$ (21).

$\mathrm{CP}$ is are a new revolutionary class of drugs, which act on PD-L1. The activation of PD-1/PD-L1 signaling let tumor cells evade the antigen-specific T-cell immunologic response. Therefore, blocking this checkpoint signaling may represent an effective cancer therapy (22). Pembrolizumab and nivolumab, the two leading drugs of the first $\mathrm{CPi}$ generation, have demonstrated substantial activity in several solid and hematological tumors and are now approved for the treatment of several malignancies $(23,24)$.

Besides the presence of 9p24.1 locus alteration, and again as also shown in HL, PD-L1 expression and clinical response of PMBCL to pembrolizumab are related to the magnitude of 9 p24 abnormality that determines the PDL-1 expression. In this line, Armand and colleagues performed a biomarker analysis through fluorescence in situ hybridization to assess changes in the gene copy number changes; a modified $\mathrm{H}$-score ranging was thus generated (1). This $\mathrm{H}$-score was defined as the product of the percentage of malignant tumor cells with membranous staining for PD$\mathrm{L} 1$, thus ranging from 0 to 100 . In the 42 assessed patients, the rate of objective response was $25 \%$ in patients with an $\mathrm{H}$-score of $0,42 \%$ for patients with a of $1-99$, and $64 \%$ for those with aPD-L1 H-score of 100. PD-L1 expression level was also associated with PFS (1).

Like HL, PMBCL expresses CD30, although more variably. The anti-CD30 antibody drug conjugate brentuximab vedotin, approved in HL, was evaluated in relapsed/refractory PMBCL during a phase II trial. Disappointingly, only a $13 \%$ rate of overall response rate was reported, $13 \%$ and the trial was thus terminated early (Table 1) (12). Conversely, when brentuximab was associated to a $\mathrm{CPi}$, the magnitude of response rates was higher than the sum of a single-agent performance, and this combination presently represents the most effective 
approach in relapsing/refractory PMBCL. In 30 patients failing at least two previous treatment lines, two-thirds of whom with refractory disease, the association of nivolumab and brentuximab vedotin induced a $73 \%$ overall response rate with a $37 \%$ rate of CR (13). Median duration of response was not reached, and $11 / 30$ patients were bridged to transplantation. The safety of this combination strategy was acceptable and no additive adverse effects of the two drugs were reported. Given the low activity of brentuximab vedotin as single agent in PMBCL, the authors of that analysis argued that in the combination setting, brentuximab probably exerts its antitumor activity by the depletion of intratumoral T-regulatory cells and the induction of an immunogenic cell death. Both these mechanisms enhance antitumor immunity, which is further boosted by PD-1 blockers (13).

Another approach to be outlined in the therapeutic armamentarium of relapsing-remitting PMBCL is the chimeric antigen receptor (CAR) $\mathrm{T}$ cell therapy $(14,25)$. In short, it consists of administration of $\mathrm{T}$ lymphocytes modified to express an anti-CD19 chimeric antigen. The outcomes of this strategy in DLBLC have been largely described in the last years $(14,25)$, showing outstanding outcome for cases with high-risk refractory disease. Encouraging data on small number of cases with PMBCL were extrapolated from larger series and further analysis are awaited from ongoing trials to establish the activity of this approach in PMBCL.

To our knowledge, CPis are single agents with the highest activity in PMBCL (Table 1). Their association with classical chemotherapy, as well as with other biological or immunological strategies, are being assessed in prospective ongoing trials at an earlier phase of the clinical history in PMBCL. These trials will obviously represent an interesting challenge in the next years, especially if an appropriate selection of truly high-risk patients will be performed. Indeed, we should not forget that about $80 \%$ of PMBCL patients can be cured with standard first-line therapy and, thus, the primary effort must focus on identifying those who probably will not. The need for a specific genetic alteration and the correlation with the amount of the molecular abnormalities reflects the current trend to stratify the choice treatment according to the biological and molecular characteristics of the disease subgroup (1).

\section{Acknowledgments}

Funding: Editorial assistance was provided by Luca
Giacomelli, PhD, and Aashni Shah (Polistudium SRL, Milan, Italy), and was supported by internal funds.

\section{Footnote}

Conflicts of Interest: Both authors have completed the ICMJE uniform disclosure form (available at http://dx.doi. org/10.21037/atm.2020.04.06). The authors have no conflicts of interest to declare.

Ethical Statement: The authors are accountable for all aspects of the work in ensuring that questions related to the accuracy or integrity of any part of the work are appropriately investigated and resolved.

Open Access Statement: This is an Open Access article distributed in accordance with the Creative Commons Attribution-NonCommercial-NoDerivs 4.0 International License (CC BY-NC-ND 4.0), which permits the noncommercial replication and distribution of the article with the strict proviso that no changes or edits are made and the original work is properly cited (including links to both the formal publication through the relevant DOI and the license). See: https://creativecommons.org/licenses/by-nc-nd/4.0/.

\section{References}

1. Armand P, Rodig S, Melnichenko V, et al. Pembrolizumab in relapsed or refractory primary mediastinal large B-cell lymphoma. J Clin Oncol 2019;37:3291-9.

2. Todeschini G, Secchi S, Morra E, et al. Primary mediastinal large B-cell lymphoma (PMLBCL): long-term results from a retrospective multicentre Italian experience in 138 patients treated with $\mathrm{CHOP}$ or MACOPB/ VACOP-B. Br J Cancer 2004;90:372-6.

3. Möller P, Moldenhauer G, Momburg F, et al. Mediastinal lymphoma of clear cell type is a tumor corresponding to terminal steps of B cell differentiation. Blood 1987;69:1087-95.

4. Bertini M, Orsucci L, Vitolo U, et al. Stage II large B-cell lymphoma with sclerosis treated with MACOP-B. Ann Oncol 1991;2:733-7.

5. Rosenwald A, Wright G, Leroy K, et al. molecular diagnosis of primary mediastinal $\mathrm{B}$ cell lymphoma identifies a clinically favorable subgroup of diffuse large B cell lymphoma related to Hodgkin lymphoma. J Exp Med 2003;198:851-62.

6. Zinzani PL, Martelli M, Bertini M, et al.; International 
Extranodal Lymphoma Study Group (IELSG). Induction chemotherapy strategies for primary mediastinal large B-cell lymphoma with sclerosis: a retrospective multinational study on 426 previously untreated patients. Haematologica 2002;87:1258-64.

7. Rieger M, Osterborg A, Pettengell R, et al.; MabThera International Trial (MInT) Group. Primary mediastinal B-cell lymphoma treated with CHOP-like chemotherapy with or without rituximab: results of the Mabthera International Trial Group study. Ann Oncol 2011;22:664-70.

8. Giulino-Roth L. How I treat primary mediastinal B-cell lymphoma. Blood. 2018;132:782-90.

9. Vardhana S, Hamlin PA, Yang J, et al. Outcomes of relapsed and refractory primary mediastinal (thymic) large B-cell lymphoma treated with second-line therapy and intent to transplant. Biol Blood Marrow Transplant 2018;24:2133-8.

10. Avivi I, Boumendil A, Finel H, et al. Autologous stem cell transplantation for primary mediastinal B cell lymphoma: long-term outcome and role of post-transplant radiotherapy. A report of the European Society for Blood and Marrow Transplantation. Bone Marrow Transplant 2018;53:1001-9.

11. Kuruvilla J, Pintilie M, Tsang R, et al. Salvage chemotherapy and autologous stem cell transplantation are inferior for relapsed or refractory primary mediastinal large B- cell lymphoma compared with diffuse large B-cell lymphoma. Leuk Lymphoma 2008;49:1329-36.

12. Younes A, Santoro A, Shipp M, et al. Nivolumab for classical Hodgkin's lymphoma after failure of both autologous stem-cell transplantation and brentuximab vedotin: a multicentre, multicohort, single-arm phase 2 trial. Lancet Oncol 2016;17:1283-94.

13. Zinzani PL, Pellegrini C, Chiappella A. Brentuzimab vedotin in relapsed primary mediastinal large B cell lymphoma: results from a phase 2 clinical trial. Blood 2017;129:2328-30.

14. Zinzani PL, Santoro A, Gritti G, et al. Nivolumab combined with bentuximab vedotin for relapsed/refractory primary mediastinal large B cell lymphoma: efficacy and safety from the phase II CheckMate 436 study. J Clin Oncol 2019;37:3081-9.

15. Aoki T, Shimada K, Suzuki R, et al. High-dose chemotherapy followed by autologous stem cell transplantation for relapsed/refractory primary mediastinal large B-cell lymphoma. Blood Cancer J 2015;5:e372.
16. Klener P Jr, Etrych T, Klener P, et al. Biological therapy of hematologic malignancies: toward a chemotherapy-free era. Curr Med Chem 2019;26:1002-18.

17. Mottok A, Hung SS, Chavez EA, et al. Integrative genomic analysis identifies key pathogenic mechanisms in primary mediastinal large-B cell lymphoma. Blood 2019;134:802-13.

18. Savage KJ, Monti S, Kutok JL, et al. The molecular signature of mediastinal large B-cell lymphoma differs from that of other diffuse large B-cell lymphomas and shares features with classical Hodgkin lymphoma. Blood 2003;102:3871-9.

19. Steidl C, Gascoyne RD. The molecular pathogenesis of primary mediastinal large B-cell lymphoma. Blood 2011;118:2659-69.

20. Twa DD, Chan FC, Ben-Neriah S, et al. Genomic rearrangements involving programmed death ligands are recurrent in primary mediastinal large B-cell lymphoma. Blood 2014;123:2062-5.

21. Shi M, Roemer MG, Chapuy B, et al. Expression of programmed cell death 1 ligand 2 (PD-L2) is a distinguishing feature of primary mediastinal (thymic) large B-cell lymphoma and associated with PDCD1LG2 copy gain. Am J Surg Pathol 2014;38:1715-23.

22. Green MR, Monti S, Rodig SJ, et al. Integrative analysis reveals selective 9p24.1 amplification, increased PD-1 ligand expression, and further induction via JAK2 in nodular sclerosing Hodgkin lymphoma and primary mediastinal large B-cell lymphoma. Blood 2010;116:3268-77.

23. Iwai $Y$, Ishida $M$, Tanaka $Y$, et al. Involvement of PD-L1 on tumor cells in the escape from host immune system and tumor immunotherapy by PD-L1 blockade. Proc Natl Acad Sci USA 2002;99:12293-7.

24. Brahmer JR, Tykodi SS, Chow LQ, et al. Safety and activity of anti-PD-L1 antibody in patients with advanced cancer. N Engl J Med 2012;366:2455-65.

25. Locke FL, Ghobadi A, Jacobson CA, et al: Long-term safety and activity of axicabtagene ciloleucel in refractory large B-cell lymphoma (ZUMA-1): a single-arm, multicentre, phase 1-2 trial. Lancet Oncol 2019;20:31-42.

Cite this article as: Balzarotti $M$, Santoro A. Checkpoint inhibitors in primary mediastinal B-cell lymphoma: a step forward in refractory/relapsing patients? Ann Transl Med 2020;8(16):1035. doi: 10.21037/atm.2020.04.06 